\title{
Bioactive Synthetic-Bioinformatic Natural Product Cyclic Peptides Inspired by Nonribosomal Peptide Synthetase Gene Clusters from the Human Microbiome
}

\author{
John Chu§, Xavier Vila-Farres§, Sean F. Brady ${ }^{\star}$ \\ Laboratory of Genetically Encoded Small Molecules, The Rockefeller University, New York, New \\ York 10065, United States
}

\begin{abstract}
Bioinformatic analysis of sequenced bacterial genomes has uncovered an increasing number of natural product biosynthetic gene clusters (BGCs) to which no known bacterial metabolite can be ascribed. One emerging method we have investigated for studying these BGCs is the syntheticBioinformatic Natural Product (syn-BNP) approach. The syn-BNP approach replaces transcription, translation, and in vivo enzymatic biosynthesis of natural products with bioinformatic algorithms to predict the output of a BGC and in vitro chemical synthesis to produce the predicted structure. Here we report on expanding the syn-BNP approach to the design and synthesis of cyclic peptides inspired by nonribosomal peptide synthetase BGCs associated with the human microbiota. While no syn-BNPs we tested inhibited the growth of bacteria or yeast, five were found to be active in the human cell-based MTT metabolic activity assay. Interestingly, active peptides were mostly inspired by BGCs found in the genomes of opportunistic pathogens that are often more commonly associated with environments outside the human microbiome. The cyclic syn-BNP studies presented here provide further evidence of its potential for identifying bioactive small molecules directly from the instructions encoded in the primary sequences of natural product BGCs.
\end{abstract}

\begin{abstract}
In recent years, tremendous resources have been allocated to the sequencing and bioinformatic analysis of bacterial genomes. These studies have uncovered a growing collection of natural product biosynthetic gene clusters (BGCs) to which no known metabolites can be assigned. ${ }^{1,2}$ As this pool of "silent" BGCs has grown, an increasing number of approaches have been investigated to access the metabolites it encodes. ${ }^{3,4} \mathrm{We}$ are exploring a cell-free discovery approach, where instead of relying on biological processes to decode genetic instructions and produce metabolites biosynthetically, we use bioinformatic algorithms to predict the molecular structure encoded by a BGC and chemical synthesis to build the predicted structure. The molecules produced in this process are not intended to be exact copies of natural products but instead close structural mimics that are called synthetic-
\end{abstract}

\footnotetext{
*Corresponding Author sbrady@ rockefeller.edu.

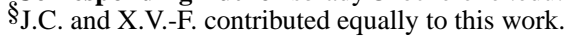

Supporting Information

The Supporting Information is available free of charge on the ACS Publications website at DOI: 10.1021/jacs.9b07317.

The authors declare no competing financial interest.
} 
Bioinformatic Natural Products (syn-BNPs). ${ }^{5,6}$ Here we use a syn-BNP approach to explore the biological activity of cyclic peptides inspired by nonribosomal peptide synthetase (NRPS) BGCs found in the human microbiome.

NRPS BGCs encode short amino acid-based natural products that are produced independent of the ribosome. ${ }^{7}$ They are particularly well suited for a large-scale discovery effort using the syn-BNP approach as bioinformatic algorithms for predicting nonribosomal peptide (NRP) structures directly from the primary sequences of NRPS genes, as well as solid-phase synthesis methods for building peptides, are both well-developed technologies. In our initial syn-BNP studies, we identified bioactive structures through the synthesis and screening of linear peptides inspired by NRPS BGCs. ${ }^{5,6,8,9}$ The efficiency of accessing bioactive small molecules from silent BGCs using the syn-BNP approach will undoubtedly improve with the introduction of increasingly sophisticated synthetic features that better mimic the structural complexity seen in natural products. NRPs are predominantly produced as cyclic structures, 10,11 and therefore we thought peptide cyclization was the most logical next level of synthetic complexity to introduce into NRPS-inspired syn-BNPs.

Uncharacterized BGCs in the human microbiome have garnered interest for their potential to encode metabolites that could shed light on how the microbiome influences its human host and eventually prove useful as therapeutics. ${ }^{5,12-17}$ Humans host hundreds of different species of bacteria, a rapidly growing percentage of which have been fully sequenced. ${ }^{18,19} \mathrm{To}$ identify starting points for the synthesis of syn-BNP cyclic peptides, we analyzed NRPS BGCs found in the genomes of 1,298 human-associated bacteria. ${ }^{18,19}$ In this survey we removed BGCs associated with known natural products as well as those that appeared to be truncated in poorly assembled genomes. As short NRPs are often highly modified, and therefore not as easily accessible through a syn-BNP approach, we also excluded BGCs containing four or fewer modules from our analysis. Ultimately, we identified 48 large ( $\geq 5$ modules) NRPS BGCs that we predicted to encode for previously uncharacterized metabolites. Among these BGCs, 25 yielded robust bioinformatic predictions that were readily compatible with solid-phase peptide synthesis (Table S1). All BGCs in this subset contained clear initiation and termination modules, appeared to be organized in an orthodox collinear fashion, and did not contain polyketide modules, heterocyclic modules, or large numbers of predicted tailoring genes. In five cases where an individual or a small number of residue predictions differed dramatically between NRP prediction algorithms, ${ }^{20-22}$ we designed two syn-BNP peptides to give 30 distinct peptide target sequences in total (Table S1).

Of the human microbiome associated BGCs we identified, more than half (27 BGCs) originated from bacteria associated with the oral microbiome (Figure 1). ${ }^{23}$ The remaining 21 BGCs were found in the genomes of bacteria isolated from the gastrointestinal tract, skin, urogenital tract, airways, and blood. The most common taxonomic source of these BGCs was Proteobacteria, followed by Actinobacteria and Firmicutes. This stands in contrast to fermentation-based discovery studies using bacteria from many other environments, where Actinobacteria have proved to be the most productive source of complex bioactive natural products. Interestingly, the number of large NRPS BGCs a taxon contributed to our study did not correlate with the number of species it commonly contributes to the human 
microbiome. For example, Bacteroidetes, one of the most common phyla in the human microbiota, did not contribute any large BGCs to this collection. As the human microbiome was predicted to encode an experimentally manageable number of unexplored target BGCs, which are potentially enriched in metabolites that interact with the human host, we felt they represented an ideal test case for expanding the syn-BNP approach to more complex cyclic structures.

While existing bioinformatic algorithms are quite good at determining the amino acid sequence of an NRP, they are not designed to predict its mode of cyclization. In canonical NRPS biosynthesis, cyclic peptides are produced by thioesterase domains that catalyze the reaction of an internal nucleophile with the $C$-terminus of the peptide. ${ }^{24}$ Three different nucleophiles are commonly used in these reactions. In the simplest case, the $N$-terminus serves as the nucleophile to form a head-to-tail cycle (Figure 2a, cHT). An amino acid sidechain can also serve as the nucleophile (Figure 2a, cSC), and in $\mathrm{N}$-acylated peptides an oxidation on the fatty acid can serve as the nucleophile (Figure 2a, cFA). Although the mode of cyclization used naturally is difficult to predict with high confidence, this was not an issue as the versatility of solid-phase peptide synthesis allowed us to synthesize all three types of cyclic peptides for each syn-BNP target (Figure 2b). ${ }^{25}$

Our analysis of known cyclic NRPs revealed that when more than one potential side-chain nucleophile is present, the majority of structures are cyclized through the residue that yielded the largest macrocycle (Figure S2). This analysis also revealed that fatty acid cyclized peptides are often cyclized through a $\beta$-oxidation on the fatty acid. Our syntheses were designed to conform to these observations. To facilitate side-chain cyclization, serine/ threonine nucleophiles were replaced with 2,3-diaminopropionic acid, an isosteric residue that contains a more reactive nucleophile $\left(\mathrm{NH}_{2}\right)$. The 2,3-diaminopropionic acid was protected with an allyloxycarbonyl group to permit its selective deprotection. Similarly, to facilitate cyclization through the fatty acid, 3-aminodecanoic acid was used in the synthesis of all fatty acid cyclized peptides.

Based on the 30 peptide sequences we predicted from 25 human NRPS BGCs, we designed 86 cyclic syn-BNP target structures, each with a distinct sequence or cyclization pattern (Table S1). Four peptides did not contain any nucleophilic amino acids for use in the synthesis of side-chain cyclized product. All syntheses were performed on 2-chlorotrityl resins using standard Fmoc/ $t \mathrm{Bu}$ building blocks. Upon completion of peptide synthesis, the resins were split and a portion was capped with $N$-Fmoc-3-aminodecanoic acid. For $N$ - to $C$ terminus and fatty acid cyclized peptides, the $N$-terminal Fmoc protecting group was removed, and for side-chain cyclized peptides the allyloxycarbonyl protecting group was removed. Peptides were then released from the resins with mild acid treatment. Activation of the $C$-terminal carboxylate to promote cyclization with the deprotected nucleophile was accomplished using PyAOP as a coupling reagent. Cyclized peptides were globally deprotected and HPLC purified prior to biological testing. After two rounds of synthesis we successfully produced 72 of 86 syn-BNP targets.

While bacterial natural products have diverse biological activities, they have frequently been associated with antibacterial, antifungal or antiproliferative activities. ${ }^{26} \mathrm{We}$ screened all syn- 
BNPs for their ability to inhibit the growth of Gram-positive bacteria (Staphylococcus aureus), Gram-negative bacteria (Escherichia coli), and fungi (Candida albicans). These peptides were also screened against HeLa cells using the MTT metabolic activity assay. In our initial single-concentration assays ( $10 \mu \mathrm{g} / \mathrm{mL}$ in quadruplicate, Figure $3 \mathrm{a})$, no syn-BNPs inhibited the growth of bacteria or fungi; however, five were active against HeLa cells based on the MTT assay (Figure 3b). ${ }^{27}$ Hits were given simple names (syn-hoagimin A and B, syn-rhodomin, syn-parascrofin, and syn-kroppenstin, Figure 4) based on the source of the BGCs that inspired them. Syn-hoagimin A and B were designed based on the same BGC and differ only in the way they were cyclized (Figure 4b). The more potent fatty acid cyclized analog, syn-hoagimin B, was used in follow-up assays. To validate the observed activity, hits were resynthesized, HPLC purified, validated through high-resolution mass spectrometry (Table S4), and retested for MTT activity.

All resynthesized peptides showed high nanomolar to micromolar $\mathrm{IC}_{50} \mathrm{~s}$ in the MTT assay using four different cell lines (HeLa, HT-29, A549, NCI-H1299, Table S3). The range in potency we observed is not surprising as a syn-BNP is not an evolutionarily optimized natural product, but instead a molecule inspired by information inferred from a BGC. As seen in our initial linear peptide syn-BNP studies, future synthetic optimization of these hits is likely to identify analogs with improved potency. ${ }^{8,9}$ The MTT assay serves as an easy screen to identify molecules that interact with human cells, whether these peptides are actual toxins or in some way perturb cellular metabolic processes remains to be seen.

Syn-rhodomin and syn-hoagimin were designed based on BGCs found in the genome of a Rhodococcus hoagii strain that was isolated from human skin (Figure 4a,b). ${ }^{28}$ Rhodococcus species were historically regarded as environmental bacteria found mostly in dry soils; however, recent studies of the human microbiome have identified them as low abundance yet common participants of the microbiota. ${ }^{29}$ They are historically well known as pathogens of domesticated animals ${ }^{30}$ and more recently as opportunistic human pathogens that pose a threat to immunocompromised individuals. ${ }^{31}$ Due to their common appearance in diverse environments, Rhodococcus species have been extensively explored for the production of bioactive natural products. ${ }^{32}$ To the best of our knowledge, no compound resembling either syn-rhodomin or syn-hoagimin was reported in these studies. In addition, our analysis of metabolites produced by $R$ hoagii in pure culture using liquid chromatography-mass spectrometry failed to identify any signals associated with peptides resembling either synrhodomin or syn-hoagimin. This was not surprising as most BGCs remain silent under laboratory conditions, which was one of the driving forces behind our development of the syn-BNP approach.

Syn-parascrofin was designed based on a BGC found in the genome of Mycobacterium parascrofulaceum (Figures 4c). M. parascrofulaceum has mostly been found in human respiratory tracts. It is one of many newly identified nontuberculous mycobacteria discovered via sequence-based methods and thought to be associated with opportunistic infections in immunocompromised patients. ${ }^{28}$ Syn-kroppenstin was inspired by a BGC found in the genome of Kroppenstedtia eburnea (Figure 4d), an aerobic spore-forming Gram-positive bacterium found in the human microbiome as well as other diverse environments, including grains, soil, compost, river water, and marine sediments. ${ }^{33}$ 
Although $K$. eburnea has been isolated from normally sterile body sites of multiple sick patients, the pathogenicity of this bacterium has yet to be definitively established. ${ }^{34}$ As was the case for syn-rhodomin and syn-hoagimin, neither syn-parascrofin nor syn-kroppenstin closely resembles any previously reported NRP structures.

Our analysis of 1298 bacterial genomes associated with the human microbiome revealed 48 large NRPS BGCs that could serve as syn-BNP targets. As a frame of reference, in a similar analysis of sequenced soil Actinomycetes, historically the most productive source of natural product-based therapeutics, we found that the same number of large NRPS BGCs could, on average, be found in as few as 30 Actinomycete genomes. Interestingly, BGCs that yielded bioactive syn-BNP cyclic peptides in this study largely arise from potential pathogens that are often associated with environments outside the human microbiome. While bacteria strictly associated with the human microbiome undoubtedly produce numerous metabolites that affect human physiology, ${ }^{35-40}$ our results suggest they may not encode a large reservoir of complex natural products, especially NRPs that resemble metabolites that have historically inspired the development of therapeutics. ${ }^{26,41}$ When tested against bacteria, fungi, and human cells, syn-BNP cyclic peptides inspired by the human microbiome exclusively showed activity in the human cell-based MTT assay, suggesting that, while the microbiota may not produce large numbers of complex NRPs, the metabolites it does encode may be evolutionarily enriched for activities that affect human cells. Additional bioactivity assays are required to elucidate the mode of action of these syn-BNP cyclic peptides.

The studies outlined here suggest that continued expansion of the synthetic and bioinformatic complexity of the syn-BNP discovery pipeline will increase the accessible pool of new bioactive small molecules that are inspired by the rapidly growing collection of uncharacterized BGCs found in sequenced bacterial genomes.

\section{Supplementary Material}

Refer to Web version on PubMed Central for supplementary material.

\section{ACKNOWLEDGMENTS}

We thank the following laboratory and resource centers at the Rockefeller University for bacterial strains and technical support: Fischetti Lab (S. aureus), High-Throughput Screening Resource Center, Spectroscopy Resource Center (NMR), and Proteomics Resource Center (HRMS). This work was supported by NIH U19AI142731 and R35GM122559.

\section{REFERENCES}

(1). Nett M; Ikeda H; Moore BS Genomic basis for natural product biosynthetic diversity in the actinomycetes. Nat. Prod. Rep 2009, 26, 1362-1384. [PubMed: 19844637]

(2). Quince C; Walker AW; Simpson JT; Loman NJ; Segata N Shotgun metagenomics, from sampling to analysis. Nat. Biotechnol 2017, 35, 833-844. [PubMed: 28898207]

(3). Hertweck C Hidden biosynthetic treasures brought to light. Nat. Chem. Biol 2009, 5, 450-452. [PubMed: 19536102]

(4). Rutledge PJ; Challis GL Discovery of microbial natural products by activation of silent biosynthetic gene clusters. Nat. Rev. Microbiol 2015, 13, 509-523. [PubMed: 26119570] 
(5). Chu J; Vila-Farres X; Inoyama D; Ternei M; Cohen LJ; Gordon EA; Reddy BV; Charlop-Powers Z; Zebroski HA; Gallardo-Macias R; Jaskowski M; Satish S; Park S; Perlin DS; Freundlich JS; Brady SF Discovery of MRSA active antibiotics using primary sequence from the human microbiome. Nat. Chem. Biol 2016, 12, 1004-1006. [PubMed: 27748750]

(6). Vila-Farres X; Chu J; Inoyama D; Ternei MA; Lemetre C; Cohen LJ; Cho W; Reddy BV; Zebroski HA; Freundlich JS; Perlin DS; Brady SF Antimicrobials inspired by nonribosomal peptide synthetase gene clusters. J. Am. Chem. Soc 2017, 139, 1404-1407. [PubMed: 28055186]

(7). Walsh CT Insights into the chemical logic and enzymatic machinery of NRPS assembly lines. Nat. Prod. Rep 2016, 33, 127-135. [PubMed: 26175103]

(8). Chu J; Vila-Farres X; Inoyama D; Gallardo-Macias R; Jaskowski M; Satish S; Freundlich JS; Brady SF Human microbiome inspired antibiotics with improved $\beta$-lactam synergy against MDR Staphylococcus aureus. ACS Infect. Dis 2018, 4, 33-38. [PubMed: 28845973]

(9). Vila-Farres X; Chu J; Ternei MA; Lemetre C; Park S; Perlin DS; Brady SF An optimized synthetic-bioinformatic natural product antibiotic sterilizes multidrug-resistant Acinetobacter baumannii-infected wounds. mSphere 2018, 3 (1), 00528-17.

(10). Sieber SA; Marahiel MA Learning from nature's drug factories: nonribosomal synthesis of macrocyclic peptides. J. Bacteriol 2003, 185, 7036-7043. [PubMed: 14645262]

(11). Horsman ME; Hari TP; Boddy CN Polyketide synthase and non-ribosomal peptide synthetase thioesterase selectivity: logic gate or a victim of fate? Nat. Prod. Rep 2016, 33, 183-202. [PubMed: 25642666]

(12). Mazmanian SK; Round JL; Kasper DL A microbial symbiosis factor prevents intestinal inflammatory disease. Nature 2008, 453, 620-625. [PubMed: 18509436]

(13). Wyatt MA; Wang W; Roux CM; Beasley FC; Heinrichs DE; Dunman PM; Magarvey NA Staphylococcus aureus nonribosomal peptide secondary metabolites regulate virulence. Science 2010, 329, 294-296. [PubMed: 20522739]

(14). Yoshimoto S; Loo TM; Atarashi K; Kanda H; Sato S; Oyadomari S; Iwakura Y; Oshima K; Morita H; Hattori M; Honda K; Ishikawa Y; Hara E; Ohtani N Obesity-induced gut microbial metabolite promotes liver cancer through senescence secretome. Nature 2013, 499, 97-101. [PubMed: 23803760]

(15). Leimena MM; Ramiro-Garcia J; Davids M; van den Bogert B; Smidt H; Smid EJ; Boekhorst J; Zoetendal EG; Schaap PJ; Kleerebezem M A comprehensive metatranscriptome analysis pipeline and its validation using human small intestine microbiota datasets. BMC Genomics 2013, 14, 530. [PubMed: 23915218]

(16). An D; Oh SF; Olszak T; Neves JF; Avci FY; Erturk-Hasdemir D; Lu X; Zeissig S; Blumberg RS; Kasper DL Sphingolipids from a symbiotic microbe regulate homeostasis of host intestinal natural killer T cells. Cell 2014, 156, 123-33. [PubMed: 24439373]

(17). Donia MS; Cimermancic P; Schulze CJ; Wieland Brown LC; Martin J; Mitreva M; Clardy J; Linington RG; Fischbach MA A systematic analysis of biosynthetic gene clusters in the human microbiome reveals a common family of antibiotics. Cell 2014, 158, 1402-1414. [PubMed: 25215495]

(18). Chen T; Yu WH; Izard J; Baranova OV; Lakshmanan A; Dewhirst FE The Human Oral Microbiome Database: a web accessible resource for investigating oral microbe taxonomic and genomic information. Database 2010, 2010, baq013. [PubMed: 20624719]

(19). Human Microbiome Project Consortium. Structure, function and diversity of the healthy human microbiome. Nature 2012, 486, 207-214. [PubMed: 22699609]

(20). Stachelhaus T; Mootz HD; Marahiel MA The specificity-conferring code of adenylation domains in nonribosomal peptide synthetases. Chem. Biol 1999, 6, 493-505. [PubMed: 10421756]

(21). Minowa Y; Araki M; Kanehisa M Comprehensive analysis of distinctive polyketide and nonribosomal peptide structural motifs encoded in microbial genomes. J. Mol. Biol 2007, 368, 1500-1517. [PubMed: 17400247]

(22). Rottig M; Medema MH; Blin K; Weber T; Rausch C; Kohlbacher O NRPSpredictor2: a web server for predicting NRPS adenylation domain specificity. Nucleic Acids Res 2011, 39 (Web Server issue), W362-W367. [PubMed: 21558170] 
(23). Dewhirst FE; Chen T; Izard J; Paster BJ; Tanner AC; Yu WH; Lakshmanan A; Wade WG The human oral microbiome. J. Bacteriol 2010, 192, 5002-5017. [PubMed: 20656903]

(24). Grunewald J; Marahiel MA Chemoenzymatic and template-directed synthesis of bioactive macrocyclic peptides. Microbiol. Mol. Biol. Rev 2006, 70, 121-146. [PubMed: 16524919]

(25). White CJ; Yudin AK Contemporary strategies for peptide macrocyclization. Nat. Chem 2011, 3 , 509-524. [PubMed: 21697871]

(26). Newman DJ; Cragg GM Natural products as sources of new drugs from 1981 to 2014. J. Nat. Prod 2016, 79, 629-661. [PubMed: 26852623]

(27). Kumar P; Nagarajan A; Uchil PD Analysis of cell viability by the MTT assay. Cold Spring Harb. Protoc 2018, 2018, 096222.

(28). Human Microbiome Reference Genome Database, https://hmpdacc.org/hmp/HMRGD/ (accessed November 2018).

(29). Kraal L; Abubucker S; Kota K; Fischbach MA; Mitreva M The prevalence of species and strains in the human microbiome: a resource for experimental efforts. PLoS One 2014, 9, No. e97279. [PubMed: 24827833]

(30). Giguere S; Cohen ND; Chaffin MK; Hines SA; Hondalus MK; Prescott JF; Slovis NM Rhodococcus equi: clinical manifestations, virulence, and immunity. J. Vet. Intern. Med 2011, 25, 1221-1230. [PubMed: 22092609]

(31). Weinstock DM; Brown AE Rhodococcus equi: an emerging pathogen. Clin. Infect. Dis 2002, 34, 1379-1385. [PubMed: 11981734]

(32). Kitagawa W; Tamura T Three types of antibiotics produced from Rhodococcus erythropolis strains. Microbes. Environ 2008, 23, 167-171. [PubMed: 21558704]

(33). von Jan M; Riegger N; Potter G; Schumann P; Verbarg S; Sproer C; Rohde M; Lauer B; Labeda DP; Klenk HP Kroppenstedtia eburnea gen. nov., sp. nov., a thermoactinomycete isolated by environmental screening, and emended description of the family Thermoactinomycetaceae Matsuo et al. 2006 emend. Yassin et al. 2009. Int. J. Syst. Evol. Microbiol 2011, 61, 2304-2310. [PubMed: 20971832]

(34). Turenne CY; Cook VJ; Burdz TV; Pauls RJ; Thibert L; Wolfe JN; Kabani A Mycobacterium parascrofulaceum sp. nov., novel slowly growing, scotochromogenic clinical isolates related to Mycobacterium simiae. Int. J. Syst. Evol. Microbiol 2004, 54, 1543-1551. [PubMed: 15388708]

(35). Cohen LJ; Esterhazy D; Kim S-H; Lemetre C; Aguilar RR; Gordon EA; Pickard AJ; Cross JR; Emiliano AB; Han SM; Chu J; Vila-Farres X; Kaplitt J; Rogoz A; Calle PY; Hunter C; Bitok JK; Brady SF Commensal bacteria make GPCR ligands that mimic human signaling molecules. Nature 2017, 549, 48-53. [PubMed: 28854168]

(36). Bansal T; Alaniz RC; Wood TK; Jayaraman A The bacterial signal indole increases epithelial cell tight-junction resistance and attenuates indicators of inflammation. Proc. Natl. Acad. Sci. U. S. A 2010, 107, 228-233. [PubMed: 19966295]

(37). Stremmel W; Schmidt KV; Schuhmann V; Kratzer F; Garbade SF; Langhans C-D; Fricker G; Okun JG Blood trimethylamine-N-oxide originates from microbiota mediated break-down of phosphatidylcholine and absorption from small intestine. PLoS One 2017, 12, No. e0170742. [PubMed: 28129384]

(38). Saha S; Rajpal DK; Brown JR Human microbial metabolite as a source of new drugs. Drug Discovery Today 2016, 21, 692-698. [PubMed: 26916596]

(39). Wilson MR; Zha L; Balskus EP Natural product discovery from the human microbiome. J. Biol. Chem 2017, 292, 8546-8552. [PubMed: 28389564]

(40). Milshteyn A; Colosimo DA; Brady SF Accessing bioactive natural products from the human microbiome. Cell Host Microbe 2018, 23, 725-736. [PubMed: 29902438]

(41). Valencia PM; Richard M; Brock J; Boglioli E The human microbiome: opportunity or hype? Nat. Rev. Drug Discovery 2017, 16, 823-824. [PubMed: 28912600] 
Human-associated bacteria $(1,000+$ genomes $)$

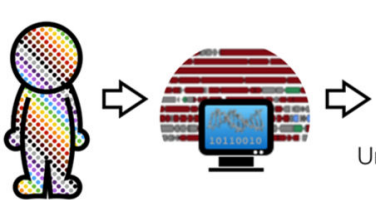
(all:suitable NRP syn-BNP targets)

Airways (2:1)

Oral (27:13)

Skin (5:5)

Urogenital (4:2)

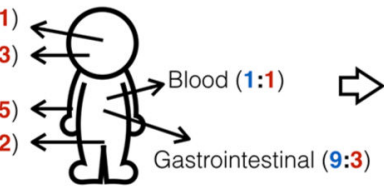

Taxa Gene clusters (all:suitable)

Actinobacteria $16: 9$

Bacteroidetes $0: 0$

Firmicutes $13: 5$

Proteobacteria 18:10

Others $1: 1$

Figure 1.

Bioinformatic analysis of the human microbiome identified 48 large NRPS BGCs. 
a)

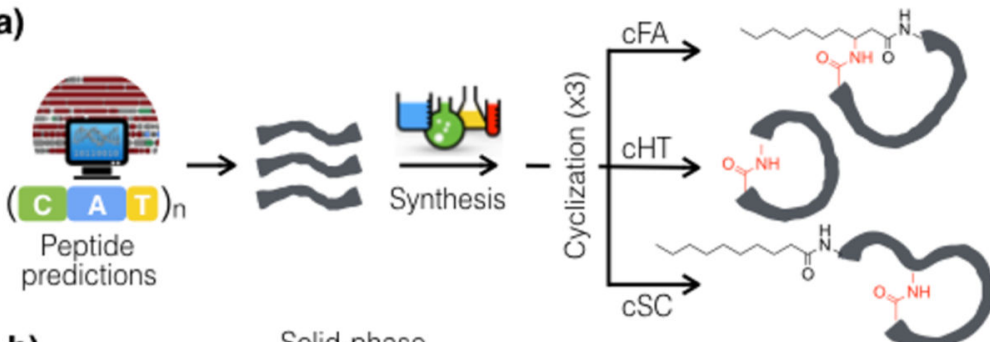

b)

Solid-phase

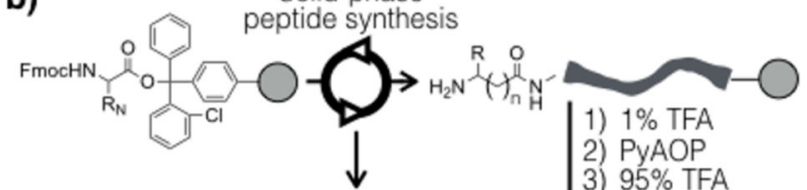

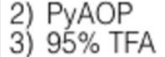
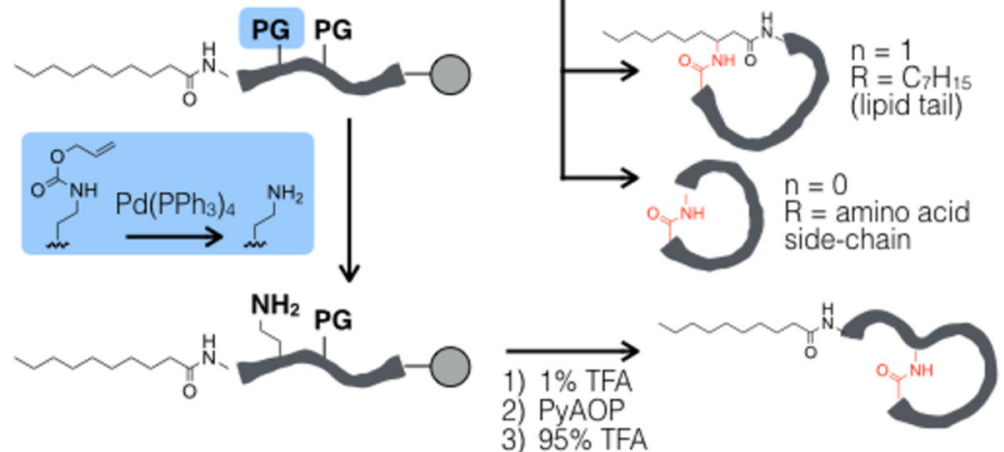

Figure 2.

(a) Peptides encoded by each of the 25 BGCs we targeted were bioinformatically predicted, chemically synthesized, and cyclized. (b) Synthetic scheme for the production of three common cyclic peptide configurations. 
a)
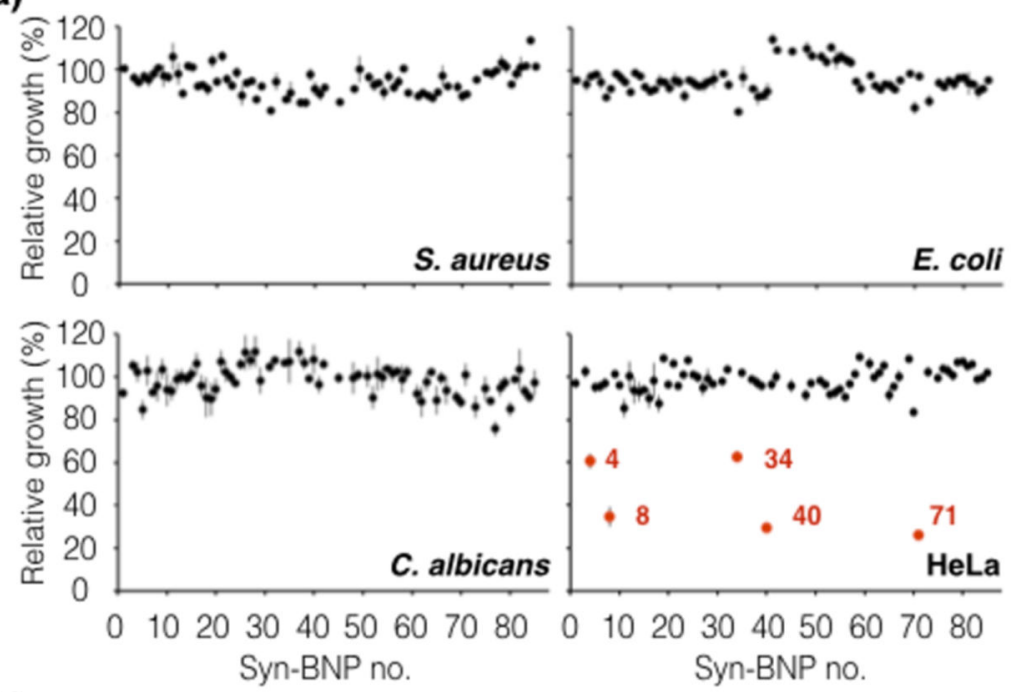

b)

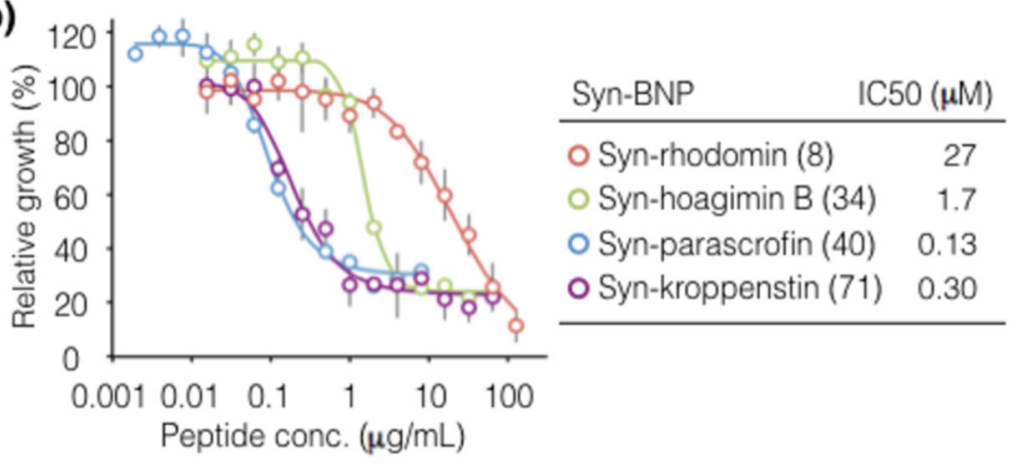

\section{Figure 3.}

(a) In the primary screen, syn-BNP cyclic peptides were tested at $10 \mu \mathrm{g} / \mathrm{mL}$ in quadruplicates, and the results are shown as percent growth relative to DMSO controls for a Gram-positive bacterium ( $S$. aureus), a Gram-negative bacterium (E. coli), a fungal pathogen (C. albicans), and a human cell line (HeLa). (b) Dose-response curves of HeLa cells measured with the MTT assay following exposure to each active syn-BNP cyclic peptide. 
a) Syn-rhodomin Structure: 6-mer, cHT peptide Species: Rhodococcus hoagii Body Site: Skin

Opportunistic pathogen

b) Syn-hoagimin $A$ and $B$

(A: $n=0, B: n=1$ )

Structure: 7-mer, cFA peptide

Species: Rhodococcus hoagii

Body Site: Skin

Opportunistic pathogen
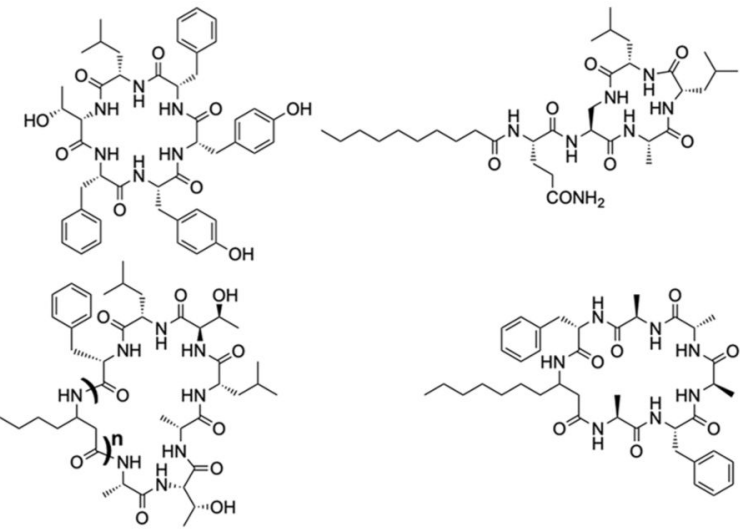

d) Syn-kroppenstin

Structure: 5-mer, cSC peptide

Species: Kroppenstedtia eburnea

Body Site: Blood

Pathogenicity to be determined

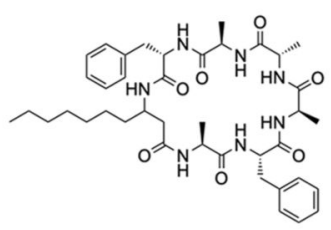

c) Syn-parascrofin

Structure: 6-mer, cFA peptide Species: Mycobacterium parascrofulaceum

Body Site: Urogenital \& respiratory tracts

Opportunistic pathogen

Figure 4.

Structure of each bioactive syn-BNP cyclic peptide and the bacterial genome from which it was predicted. 